\title{
A Novel Optimized Nonlinear Grey Bernoulli Model for Forecasting China's GDP
}

\author{
Wen-Ze Wu $\mathbb{D}^{1}{ }^{1}$ Tao Zhang, ${ }^{2}$ and Chengli Zheng $\mathbb{D}^{1}$ \\ ${ }^{1}$ School of Economics and Business Administration, Central China Normal University, Wuhan 430079, China \\ ${ }^{2}$ School of Science, Guangxi University of Science and Technology, Liuzhou 545006, China \\ Correspondence should be addressed to Wen-Ze Wu; bsstatistics@126.com
}

Received 20 February 2019; Revised 17 August 2019; Accepted 6 September 2019; Published 28 October 2019

Academic Editor: Danilo Comminiello

Copyright (c) 2019 Wen-Ze Wu et al. This is an open access article distributed under the Creative Commons Attribution License, which permits unrestricted use, distribution, and reproduction in any medium, provided the original work is properly cited.

\begin{abstract}
The nonlinear grey Bernoulli model, abbreviated as $\operatorname{NGBM}(1,1)$, has been successfully applied to control, prediction, and decisionmaking fields, especially in the prediction of nonlinear small sample time series. However, there are still some problems in improving the prediction accuracy of $\operatorname{NGBM}(1,1)$. In this paper, we propose a novel optimized nonlinear grey Bernoulli model for forecasting China's GDP. In the new model, the structure and parameters of $\operatorname{NGBM}(1,1)$ are optimized simultaneously. Especially, the latest item of first-order accumulative generating operator (1-AGO) sequence is taken as the initial condition, then background value is reconstructed by optimizing weights of neighbor values in 1-AGO sequence, which is based on minimizing the sum of absolute percentage errors, and finally, we establish the new model based on the rolling mechanism. Prediction accuracy of the proposed model is investigated through some simulations and a real example application, and the proposed model is applied to forecast the annual GDP in China from 2019 to 2023.
\end{abstract}

\section{Introduction}

Macroeconomic monitoring, forecasting, and early warning are important branches of economic research and an essential prerequisite for scientific macroeconomic decisionmaking. Among the many macroeconomic indicators, the status of GDP is the most prominent. As an important indicator to measure the size of a country's economy and market, it is the sum of values of all final products and services in a country or region for a certain period of time. Moreover, the growth rate of GDP can also reflect the growth of the country's economy and national strength to a certain extent. Thus, accurately forecasting GDP data has been a hot topic in recent years.

Annual China's GDP data should be regarded as a dynamic, nonlinear, and uncertain time series. According to the previous studies, many methods are used to predict time series problems. In general, these models can be divided into three categories, including the statistical model, intelligent model, and grey system theory. The statistical model includes linear regression [1], semiparametric and partially varying linear regression [2], nonlinear regression [3], and autoregressive moving average model (ARMA) [4]. The disadvantage of statistical models is that they rely too much on collecting data and estimating parameters. As for the intelligent model, such as artificial neural network [5] and support vector machine [6], the common drawback is that it requires lots of data to train the model. Grey system theory, firstly proposed by Deng [7], is developed to solve uncertain and unknown system problems; the grey prediction model is often named as GM based on grey system theory. Over three decades of development, the grey model has been widely studied, for example, according to disadvantages of the traditional self-adaptive intelligence grey predictive model (SAIGM), Zeng and Liu [8] proposed a novel SAIGM model with the fractional order accumulating operator called SAIGM-FO. In 2018, Zeng and $\mathrm{Li}$ [9] proposed and optimized a new unbiased grey prediction model 
(UGM(1,1)) based on extremely limited data on the output of shale gas. Some cutting-edge research can be seen in the studies by Xie et al. [10, 11], Ma et al. [12-14], Zeng et al. $[9,15,16]$, and other scholars. The new research results for grey prediction models appear continuously in the existing literature.

However, most of these models, considered as singlevariant prediction model, are not suitable for time sequences with nonlinear characteristics, which can be obviously seen from their easy mathematical calculation [17] and first-order ordinary linear AGO; Xie and Liu [18] also found that the traditional grey model obtains a satisfactory result in the pure index sequences. To this end, professor Liu et al. [19] introduced a power exponent throughout the Bernoulli differential equation, but he only discussed a special case for the power exponent which is equal to 2, so this model is often called the grey Verhulst model. To futher expand applicability of the $\operatorname{GBM}(1,1)$ model, Chen [20] proposed a novel optimized $\operatorname{GBM}(1,1)$ model termed $\operatorname{NGBM}(1,1)$; in his research, the power index can be changed and adapted according to real-time sequence and determined by a computer program. Wang et al. [21] proposed an optimized $\operatorname{NGBM}(1,1)$ for forecasting the qualified discharge rate of industrial wastewater in China. He improved prediction precision by optimizing weighted parameters of background value in 2011. In addition, Pei et al. [22] proposed a new method combined with nonlinear squares (NLS) and nonlinear grey Bernoulli model. Wang [23] built a weighted nonlinear grey Bernoulli model (short for WNNGBM) for forecasting nonlinear economic time series with small datasets. To predict quarterly sales volume of the new energy vehicles industry from China, Pei and Li [24] put forward a data-grouping approach based on the $\operatorname{NGBM}(1,1)$ model. These models mainly discussed optimization of $\operatorname{NGBM}(1,1)$ model parameters. At the same time, the Nash NGBM $(1,1)$ model, proposed by Chen et al. [25], simultaneously optimized background value and the power index. Since then, some scholars tried to optimize the $\operatorname{NGBM}(1,1)$ model through grey differential equation; for example, Liu and Xie [26] introduced Weibull cumulative distribution function into the $\operatorname{NGBM}(1,1)$ model, the proposed model is abbreviated as $\operatorname{WBGM}(1,1)$, and they also provided the new model has the advantage of $\operatorname{NGBM}(1,1)$ and Weibull cumulative distribution. To build a more general nonlinear grey forecasting model, Ma et al. [27] proposed a novel multivariate nonlinear grey Bernoulli model (NGBMC $(1, n))$, which can be considered as a combination of the $\operatorname{NGBM}(1,1)$ and the $\operatorname{GMC}(1, n)$ with different power parameters. More recent research results can be seen in [28-31].

Although there are many obvious advantages among these models, it is easy to generate unaccepted error among these models in fact. To capture the nonlinear trend in annual GDP data from China and obtain an appreciate prediction accuracy, this paper proposes a novel optimized nonlinear grey Bernoulli model, and the main contributions can be summarized as follows:
(1) The structure and parameters of the grey prediction model are optimized simultaneously in this paper. The latest item of 1-AGO sequence is taken as the initial condition, and the weighted of neighbor values in background value is determined by minimizing the sum of absolute percentage error.

(2) The grey model is established based on the rolling mechanism, according to the principle of priority of use of new information.

(3) The validity of this proposed model is verified by numerical examples and applied to forecast China's annual GDP.

The rest of paper is organized as follows. In Section 2, we give a brief description of the $\operatorname{NGBM}(1,1)$ model. Section 3 introduces the novel optimized $\operatorname{NGBM}(1,1)$ model in detail. In Section 4, some simulations are conducted to verify the effectiveness of the proposed model. Section 5 compares the proposed model with others and applies the proposed model to predict GDP data from China in the next years. Section 6 gives some main conclusions and future work. The $\mathrm{R}$ program for simulation and prediction is listed in Algorithm 1.

\section{The Description of the Existing $\operatorname{NGBM}(1,1)$ Model}

In this section, we shall give a brief introduction of the $\operatorname{NGBM}(1,1)$ model. To adjust the traditional grey model to obtain the higher prediction precision, professor Chen [20] firstly proposes the $\operatorname{NGBM}(1,1)$ model; the modeling procedure of $\operatorname{NGBM}(1,1)$ model is described as follows.

Suppose

$$
X^{(0)}=\left\{x^{(0)}(1), x^{(0)}(2), \ldots, x^{(0)}(m)\right\}, \quad m \geq 4,
$$

is a nonnegative time sequence.

$X^{(1)}$ is constructed by the first-order accumulative generating operator, which is given by

$$
X^{(1)}=\left\{x^{(1)}(1), x^{(1)}(2), \ldots, x^{(1)}(m)\right\},
$$

where $x^{(1)}(k)=\sum_{i=1}^{k} x^{(0)}(i), k=1,2, \ldots, m$.

The equation

$$
x^{(0)}(k)+a z^{(1)}(k)=b\left[z^{(1)}(k)\right]^{n},
$$

is called the basic grey differential equation of the $\operatorname{NGBM}(1,1)$ model, where $z^{(1)}(k)=0.5 \times\left(x^{(1)}(k)+\right.$ $\left.x^{(1)}(k-1)\right)$ is defined as the background value, and $n$ is often called the power index.

The equation

$$
\frac{d x^{(1)}(t)}{d t}+a x^{(1)}(t)=b\left[x^{(1)}(t)\right]^{n}
$$

is called the whiten differential equation of the $\operatorname{NGBM}(1,1)$ model. 
\#R program of simulation and prediction in the original $N G B M(1,1)$ model raw_data $<-c(53.8580,59.2963,64.1280,68.5992,74.0060,85.0754,90.0309)$ $x<-$ raw_data [1:5] \#training data

$y<-x[-1] \# Y$

$m<-$ length $(\mathrm{x})$

ago $<-\operatorname{cumsum}(x) \# 1-A G O$ sequence

$B<-\operatorname{matrix}($ nrow $=m-1, \mathbf{n c o l}=2)$

$n<-\mathbf{s e q}(-1,0.99,0.01)$ \#Given range of parameter $n$

\#compute all errors from different $n$

err $<-$ NULL

for $(i$ in 1 :length $(n))\{$

for $(j$ in $1: \operatorname{dim}(B)[1])\{$

$B[j, 1]=-0.5 *(\operatorname{ago}[j]+\operatorname{ago}[j+1])$

$\mathrm{B}[j, 2]=(0.5 *(\operatorname{ago}[j]+\operatorname{ago}[j+1]))^{\wedge} n[i]$

\}\#construct matrix $B$

$a<-($ solve $(\mathbf{t}(B) \% * \% B) \% * \% \mathbf{t}(B) \% * \% y)$ [1].

$b<-(\operatorname{solve}(\mathbf{t}(B) \% * \% B) \% * \% \mathbf{t}(B) \% * \% y)[2]$.

$x \_$simu $<-$NULL

for $(k$ in $1: m)\{$

$x \_\operatorname{simu}[k]=\left(b / \mathrm{a}+\left(x[1]^{\wedge}(1-n[i])-b / a\right) * \exp (-(1-n[i]) *\right.$

$a *(k-1)))^{\wedge}(1 /(1-n[i]))$

\}

\#Compute the sum of absolute percentage errors

$\operatorname{err}[i]<-\operatorname{sum}\left(\operatorname{abs}\left(\operatorname{diff}\left(x \_\operatorname{simu}\right)-x[-1]\right) / x[-1]\right)$

\}

$n=n[$ which.min(err)] \#Optimal parameter $n$

for $(i$ in $1: \operatorname{dim}(B)[1])\{$

$B[i, 1]<--0.5 *(\operatorname{ago}[j]+\operatorname{ago}[j+1])$

$B[i, 2]<-(0.5 *(\operatorname{ago}[j]+\operatorname{ago}[j+1]))^{\wedge} n$

\}

$a<-(\operatorname{solve}(\mathbf{t}(B) \% * \% B) \% * \% \mathbf{t}(B) \% * \% y)$ [1] \#parameter $a$

$b<-(\operatorname{solve}(\mathbf{t}(B) \% * \% B) \% * \% \mathbf{t}(B) \% * \% y)$ [2] \#parameter $b$

for $(k$ in $1:(m+2))\{$

$x \_\operatorname{simu}[k]=\left(b / a+\left(x[1]^{\wedge}(1-n)-b / a\right) * \exp (-(1-n) * a *(k-1))\right)^{\wedge}(1 /(1-n))$

$x \_$pre $<-$diff $\left(x \_\right.$simu $)$\#predicted values

\#compute error

$\mathrm{RPE}<-\left(x \_\right.$pre-raw_data $\left.[-1]\right) /$ raw_data $[-1]$

MAPE <- sum $\left(\right.$ abs $\left(x \_p r e-r a w \_d a t a[-1]\right) /$ raw_data[-1])/(length(raw_data $\left.)-1\right)$

\#R program of the optimized $N G B M(1,1)$ model

raw_data $<-c(53.8580,59.2963,64.1280,68.5992,74.0060,85.0754,90.0309)$

$x<-$ raw_data[1:5] \#training data \& first rolling

$\# x<-$ raw_data[2:6] \#training data \& second rolling

$y<-x[-1] \# Y$

$m<-$ length $(x)$

ago $<-\operatorname{cumsum}(x) \# 1-A G O$ sequence

$B<-$ matrix $($ nrow $=m-1, \mathbf{n c o l}=2)$

$n<-\mathbf{s e q}(-1,0.99,0.01)$ \#Given range of parameter $n$

alpha $<-\mathbf{s e q}(0,1,0.01)$ \#Given range of parameter alpha

\#compute all errors from different $n$ and alpha

matri_err $<-$ list ()

for $(i$ in 1:length $(n))\{$

err $<-$ NULL

for $(j$ in $1:$ length(alpha $))\{$

for $(k$ in $1: \operatorname{dim}(B)[1])$

$B[k, 1]=-\operatorname{alpha}[j] *(\operatorname{ago}[k]+\operatorname{ago}[k+1])$

$B[k, 2]=(\operatorname{alpha}[j] *(\operatorname{ago}[k]+\operatorname{ago}[k+1]))^{\wedge} n[i]$

\}$\#$ construct matrix $B$

$a<-(\operatorname{solve}(\mathbf{t}(B) \% * \% \mathrm{~B}) \% * \% \mathbf{t}(B) \% * \% y)[1]$.

$b<-(\operatorname{solve}(\mathbf{t}(B) \% * \% B) \% * \% \mathbf{t}(B) \% * \% y)$ [2].

$x \_$simu $<-$NULL

Algorithm 1: Continued. 


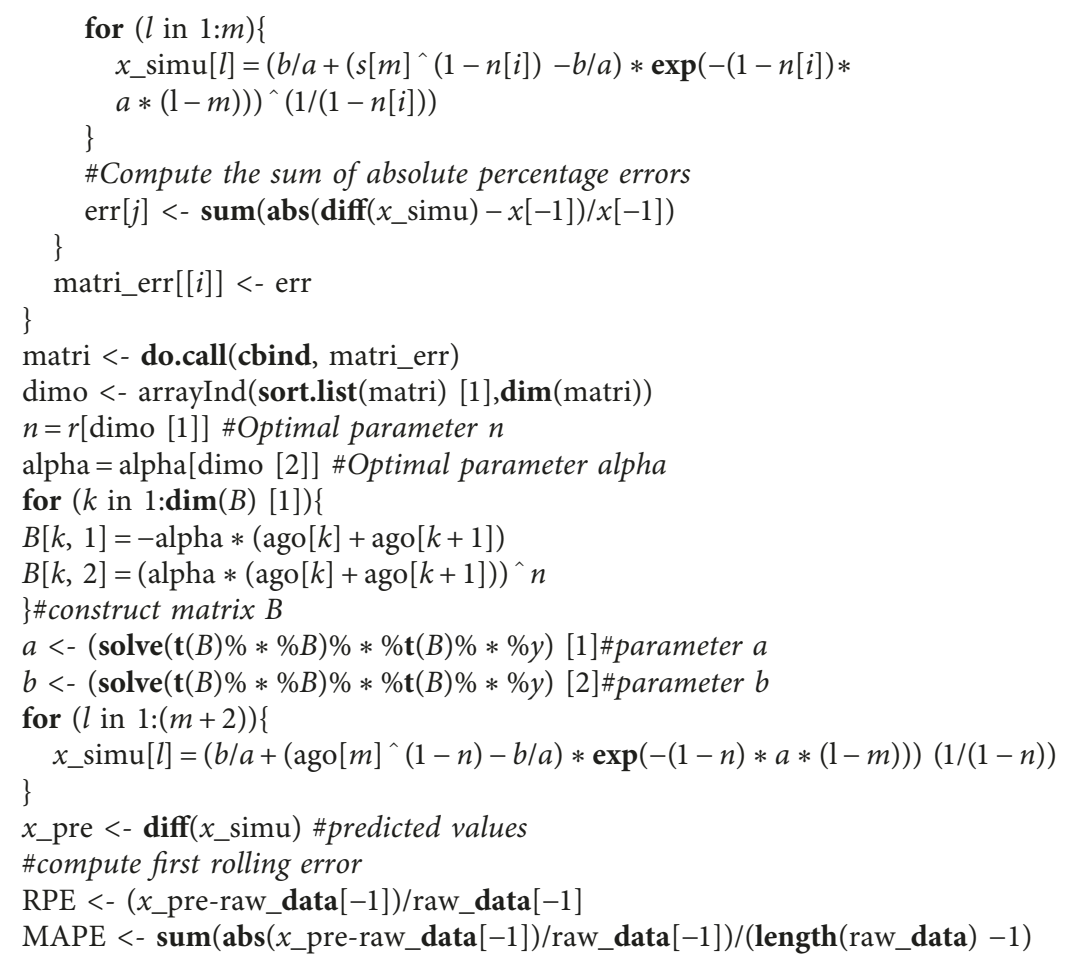

Algorithm 1

If we let

$$
\begin{aligned}
& B=\left[\begin{array}{cc}
-z^{(1)}(2) & {\left[z^{(1)}(2)\right]^{n}} \\
-z^{(1)}(3) & {\left[z^{(1)}(3)\right]^{n}} \\
\vdots & \vdots \\
-z^{(1)}(m) & {\left[z^{(1)}(m)\right]^{n}}
\end{array}\right] \\
& Y=\left[\begin{array}{c}
x^{(0)}(2) \\
x^{(0)}(3) \\
\vdots \\
x^{(0)}(m)
\end{array}\right],
\end{aligned}
$$

the model parameters $a$ and $b$ can be estimated by the least square method, which is

$$
[a, b]^{T}=\left(B^{T} B\right)^{-1} B^{T} Y \text {. }
$$
as

The general solution of $\operatorname{NGBM}(1,1)$ can be easily written

$$
x^{(1)}(t)=\left(C e^{-a(1-n) t}+\frac{b}{a}\right)^{1 /(1-n)},
$$

where $C$ is an arbitrary constant. We set $x^{(1)}(1)=x^{(0)}(1)$ and $t=1$, then we have

$$
C=\left(x^{(0)}(1)^{1-n}-\frac{b}{a}\right) e^{a(1-n)}
$$

Substituting (8) into (7), the time response function of the $\operatorname{NGBM}(1,1)$ model is given by

$$
x^{(1)}(t)=\left\{\left(x^{(0)}(1)^{1-n}-\frac{b}{a}\right)\left(e^{-a(1-n)(1-t)}+\frac{b}{a}\right)\right\}^{1 /(1-n)} .
$$

To further acquire the discrete time response function, we set $k=t$, then we have

$$
\begin{array}{r}
\widehat{x}^{(1)}(k)=\left\{\left(x^{(0)}(1)^{1-n}-\frac{b}{a}\right)\left(e^{-a(1-n)(1-k)}+\frac{b}{a}\right)\right\}^{1 /(1-n)}, \\
n \neq 1, k=1,2, \ldots .
\end{array}
$$

The predicted value of $x^{(0)}(k)$ is computed by the firstorder inverse accumulative generating operator (short for IAGO), which is

$$
\widehat{x}^{(0)}(k)=\hat{x}^{(1)}(k)-\hat{x}^{(1)}(k-1) .
$$

\section{The Optimized NGBM(1,1) Model}

According to previous studies, related to grey forecasting model, it is shown that both structure and parameters have great influence on prediction performance. For this purpose, we propose a novel optimized nonlinear grey Bernoulli model based on the rolling mechanism. 
3.1. Optimization of the Initial Condition. In the existing $\operatorname{NGBM}(1,1)$ model, the initial condition is set to be $x^{(0)}(1)$, the oldest data in the original sequence, this means all of the information expect first item is not fully used for the forecasting model. According to the principle of new information prior using, we choose the latest item of 1-AGO $x^{(1)}(m)$ as the new initial condition.

Theorem 1. Assuming $B, Y$, and $[a, b]^{T}$ are same as mentioned in Section 2, the following conclusions can be summarized:

(1) The solution of the whiten grey differential equation of the $\operatorname{NGBM}(1,1)$ model can be written as

$$
x^{(1)}(t)=\left\{\left(x^{(1)}(m)^{1-n}-\frac{b}{a}\right)\left(e^{-a(1-n)(t-m)}+\frac{b}{a}\right)\right\}^{1 /(1-n)} .
$$

(2) The discrete time response function of the $\operatorname{NGBM}(1,1)$ model is given by

$$
\widehat{x}^{(1)}(k)=\left\{\left(x^{(1)}(m)^{1-n}-\frac{b}{a}\right)\left(e^{-a(1-n)(k-m)}+\frac{b}{a}\right)\right\}^{1 /(1-n)} .
$$

Proof. Firstly, we consider the general solution of the $\operatorname{NGBM}(1,1)$ model

$$
x^{(1)}(t)=\left(C e^{-a(1-n) t}+\frac{b}{a}\right)^{1 /(1-n)} .
$$

By setting $t=m$, we obtain

$$
x^{(1)}(m)=\left(C e^{-a(1-n) m}+\frac{b}{a}\right)^{1 /(1-n)} .
$$

Then we have

$$
C=\left(x^{(1)}(m)^{1-n}-\frac{b}{a}\right) e^{a(1-n) m}
$$

Thus, the time response equation is written as

$$
\widehat{x}^{(1)}(t)=\left[\left(x^{(1)}(m)^{1-n}-\frac{b}{a}\right) e^{-a(1-n)(t-m)}+\frac{b}{a}\right]^{1 /(1-n)} \text {. }
$$

By letting $t=k$, it is easy to yield

$$
\widehat{x}^{(1)}(k)=\left[\left(x^{(1)}(m)^{1-n}-\frac{b}{a}\right) e^{-a(1-n)(k-m)}+\frac{b}{a}\right]^{1 /(1-n)} \text {. }
$$

Theorem 1 is then proved.

3.2. Optimization of Parameters. According to Wang et al. [21], it is obvious that prediction precision of the $\operatorname{NGBM}(1,1)$ model is directly influenced by the parameter $\alpha$ in the background value. The background value of both the GM $(1,1)$ model and the other derived grey model are often considered as the approximate value of the true one representing the area of the integral region between the curve and the abscissa axis in the interval $[k-1, k]$, which is mathematically expressed as

$$
z^{(1)}(k)=\int_{k-1}^{k} x^{(1)} d x .
$$

According to the mean value theory, we have

$$
z^{(1)}(k)=\alpha x^{(1)}(k)+(1-\alpha) x^{(1)}(k-1),
$$

where $\alpha \in[0,1]$, generally, $\alpha=0.5$ in the $\operatorname{GM}(1,1)$ model and its derived model. The predicted values, however, deviate from actual values in the original time sequence with large fluctuations. Meaning prediction accuracy of these models loses effectiveness in strongly fluctuating sequences.

Here, the model parameters $a$ and $b$ can be estimated with the unknown $\alpha$ by least square method, which can be written as

$$
\begin{aligned}
& {[a, b]^{T}=\left(B^{T} B\right)^{-1} B^{T} Y=\frac{1}{\sum_{k=2}^{m}\left(z^{(1)}(k)\right)^{2 n} \sum_{k=2}^{m}\left(z^{(1)}(k)\right)^{2}-\left(\sum_{k=2}^{m}\left(z^{(1)}(k)\right)^{n+1}\right)^{2}}} \\
& \times\left[\begin{array}{ll}
\sum_{k=2}^{m}\left[z^{(1)}(k)\right]^{2 n} & \sum_{k=2}^{m}\left[z^{(1)}(k)\right]^{n+1} \\
\sum_{k=2}^{m}\left[z^{(1)}(k)\right]^{n+1} & \sum_{k=2}^{m}\left[z^{(1)}(k)\right]^{2}
\end{array}\right]\left[\begin{array}{c}
-\sum_{k=2}^{m} z^{(1)}(k) x^{(0)}(k) \\
\sum_{k=2}^{m}\left(z^{(1)}(k)\right)^{n} x^{(0)}(k)
\end{array}\right] .
\end{aligned}
$$


After simplification, we have

$$
[a, b]^{T}=\left[\begin{array}{c}
\frac{\sum_{k=2}^{m}\left(z^{(1)}(k)\right)^{n+1} \sum_{k=2}^{m}\left(z^{(1)}(k)\right)^{n} x^{(0)}(k)-\sum_{k=2}^{m}\left(z^{(1)}(k)\right)^{2 n} \sum_{k=2}^{m} z^{(1)}(k) x^{(0)}(k)}{\sum_{k=2}^{m}\left(z^{(1)}(k)\right)^{2 n} \sum_{k=2}^{m}\left(z^{(1)}(k)\right)^{2}-\left(\sum_{k=2}^{m}\left(z^{(1)}(k)\right)^{n+1}\right)^{2}} \\
\frac{\sum_{k=2}^{m}\left(z^{(1)}(k)\right)^{2} \sum_{k=2}^{m}\left(z^{(1)}(k)\right)^{n} x^{(0)}(k)-\sum_{k=2}^{m}\left(z^{(1)}(k)\right)^{n+1} \sum_{k=2}^{m} z^{(1)}(k) x^{(0)}(k)}{\sum_{k=2}^{m}\left(z^{(1)}(k)\right)^{2 n} \sum_{k=2}^{m}\left(z^{(1)}(k)\right)^{2}-\left(\sum_{k=2}^{m}\left(z^{(1)}(k)\right)^{n+1}\right)^{2}}
\end{array}\right] .
$$

Thus, $a$ and $b$ are given as

$$
\begin{gathered}
a=\frac{\sum_{k=2}^{m}\left(z^{(1)}(k)\right)^{n+1} \sum_{k=2}^{m}\left(z^{(1)}(k)\right)^{n} x^{(0)}(k)-\sum_{k=2}^{m}\left(z^{(1)}(k)\right)^{2 n} \sum_{k=2}^{m} z^{(1)}(k) x^{(0)}(k)}{\sum_{k=2}^{m}\left(z^{(1)}(k)\right)^{2 n} \sum_{k=2}^{m}\left(z^{(1)}(k)\right)^{2}-\left(\sum_{k=2}^{m}\left(z^{(1)}(k)\right)^{n+1}\right)^{2}}, \\
b=\frac{\sum_{k=2}^{m}\left(z^{(1)}(k)\right)^{2} \sum_{k=2}^{m}\left(z^{(1)}(k)\right)^{n} x^{(0)}(k)-\sum_{k=2}^{m}\left(z^{(1)}(k)\right)^{n+1} \sum_{k=2}^{m} z^{(1)}(k) x^{(0)}(k)}{\sum_{k=2}^{m}\left(z^{(1)}(k)\right)^{2 n} \sum_{k=2}^{m}\left(z^{(1)}(k)\right)^{2}-\left(\sum_{k=2}^{m}\left(z^{(1)}(k)\right)^{n+1}\right)^{2}} .
\end{gathered}
$$

In this study, the parameter $\alpha$ is automatically determined by minimizing the sum of mean absolute percentage error generated from the $\operatorname{NGBM}(1,1)$ model, which can be considered as the optimization problem, we thus have

$$
\operatorname{minimize} \sum_{k=1}^{n}\left|\frac{\widehat{x}^{(0)}(k)-x^{(0)}(k)}{x^{(0)}(k)}\right| \text {, }
$$

where $x^{(0)}(k)$ and $x^{(0)}(k)$ are the actual values at time $k$ and corresponding prediction, respectively.

In the $\operatorname{NGBM}(1,1)$ model, the whole datasets are used for prediction. However, constructing the model based on the rolling mechanism has been widely employed into various grey models in recent years, which can be seen in $[32,33]$. In the proposed NGBM $(1,1)$ model, $\left\{x^{(0)}(1), x^{(0)}(2), \ldots, x^{(0)}(n)\right\}$ is used for forecasting $x^{(0)}(n+1)$, when prediction $\widehat{x}^{(0)}(n)$ is found; the oldest data $x^{(0)}(1)$ shall be deleted and the latest data $x^{(0)}(n+1)$ should be added into the modeling data, that is, $\left\{x^{(0)}(2), x^{(0)}(3), \ldots, x^{(0)}(n+1)\right\}$ is used for forecasting $x^{(0)}(n+2)$. Repeat the above procedure to find all the data.

3.3. Modeling Evaluation Criteria and Detailed Modeling Procedures. In order to compare the proposed model with other commonly used grey models, the three statistical indicators are collected for this study, including relative percentage error (RPE) and mean absolute percentage error (MAPE), which are defined as

$$
\begin{aligned}
\mathrm{RPE}_{k} & =\frac{\widehat{x}^{(0)}(k)-x^{(0)}(k)}{x^{(0)}(k)} \times 100 \%, \\
\mathrm{MAPE} & =\frac{1}{m-1} \sum_{k=2}^{m}\left|\frac{\widehat{x}^{(0)}(k)-x^{(0)}(k)}{x^{(0)}(k)}\right| \times 100 \%,
\end{aligned}
$$

respectively. In addition, the criteria of MAPE for prediction error are shown in Table 1.

Computational steps of the optimized $\operatorname{NGBM}(1,1)$ model can be summarized as follows:

Step 1. Given the original time sequence $\left\{x^{(0)}(1), x^{(0)}(2), \ldots, x^{(0)}(m)\right\}$, calculate the first-order accumulative generating operator sequence $X^{(1)}$ using (2).

Step 2. Construct the matrices $B$ and $Y$ and estimate the parameters $a$ and $b$ especially with unknown coefficient $\alpha$ using (5).

Step 3. Substitute $a$ and $b$ into (12), find the optimal parameters $\alpha$ and power index $n$ using (17).

Step 4. Re-estimate the parameters $a$ and $b$ using (5). Step 5. Compute $\widehat{X}^{(1)}$.

Step 6. According to the first-order inverse accumulative generating operator, compute the predicted value $\hat{x}^{(0)}$ using (10).

\section{Verification of the Optimized $\operatorname{NGBM}(1,1)$ Model}

To demonstrate prediction precision of the proposed model, we also establish the traditional $\operatorname{NGBM}(1,1)$ model, and the output value of optoelectronic components and applications in Taiwan are taken as examples in this section. More details are available in $[4,17]$.

The parameters $a, b, n$, and $\alpha$ of the two models shall be estimated by employing the least square method and by minimizing the sum of absolute percentage errors, respectively. $n$ is empirically provided in $[-1,0.99]$. The track of seeking parameters is detailedly shown in Figure 1, and 
TABLE 1: The MAPE criteria of prediction precision.

\begin{tabular}{lcccc}
\hline MAPE (\%) & $\leq 10$ & $10-20$ & $20-50$ & $\geq 50$ \\
\hline $\begin{array}{l}\text { Prediction } \\
\text { precision }\end{array}$ & Excellent & Good & Reasonable & Unacceptable \\
\hline
\end{tabular}

the simulative results of the two models are listed in Tables 2 and 3 .

It is a remarkable fact that, in Figure 1, both $n$ and $\alpha$ will lead the sum of absolute percentage errors to be missing or unacceptably large (e.g., $4.6 \times 10^{32}$ ) in extreme cases. To ensure the graph is clear and smooth, we set the absolute percentage error as 1 when it is missing, and we set the absolute percentage error as 10 when it is larger than 10 .

According to Table 2, the optimized $\operatorname{NGBM}(1,1)$ model with $n=-0.45, \alpha=0.46$ has a better prediction performance than that of the original $\operatorname{NGBM}(1,1)$ model in optoelectronic components. According to Table 3, prediction accuracy of the optimized $\operatorname{NGBM}(1,1)$ model with $n=0.91, \alpha=0.46$ is higher than that of the $\operatorname{NGBM}(1,1)$ model in optoelectronic application. In the optimized $\operatorname{NGBM}(1,1)$ model, MAPE are reduced from $4.82 \%$ and $4.10 \%$ to $4.28 \%$ and $3.64 \%$, meaning the optimized $\operatorname{NGBM}(1,1)$ model is more effective and applicable than the original $\operatorname{NGBM}(1,1)$ model.

\section{Application}

5.1. Data Source. In this section, we consider the annual GDP data from China, which can be downloaded from the official website of the National Bureau of Statistics of China (http://www.stats.gov.cn/English/) as listed in Table 4. The data from 2012 to 2016 are used for train models, and data from 2017 to 2018 are used for testing models.

5.2. Forecasting Results. To further demonstrate effectiveness and applicability of the optimized $\operatorname{NGBM}(1,1)$ model (short for the new model), we also establish the other comparative models, including the $\operatorname{NGBM}(1,1)$ model (written as $\mathrm{M} 1$ ), $\operatorname{NGBM}(1,1)$ model of initial condition optimization (written as $M 2), \operatorname{NGBM}(1,1)$ model of background value optimization (written as M3), and $\operatorname{NGBM}(1,1)$ model of initial condition and background value optimization without the rolling mechanism (written as M4).

In M1 and M2, the unknown parameters include $a, b$, and $n$. In M3, M4, and the new model, in addition to the parameters above, there is an unknown parameter $\alpha$. By calculating, the model parameters of the five models are shown in Table 5, and results of simulation and prediction are listed in Tables 6 and 7; the results of simulation and prediction also can be clearly seen in Figure 2. The $\mathrm{R}$ program for calculating model parameters, simulating, and predicting results are shown in Algorithm 1.

According to Tables 6 and 7, we can easily observe the five grey models are basically close to the actual values in training period, that is, the relative error values generated

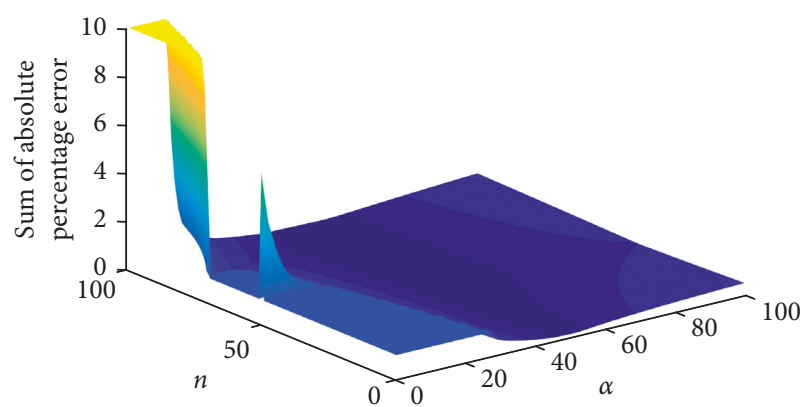

FIGURE 1: The track of simultaneously seeking the parameters $n$ and $\alpha$ in the optoelectronic components.

TABle 2: Results of optoelectronic components from the two models.

\begin{tabular}{|c|c|c|c|c|c|}
\hline \multirow[t]{2}{*}{ Year } & \multirow[t]{2}{*}{ Actual data } & \multicolumn{2}{|c|}{$\begin{array}{c}\operatorname{NGBM}(1,1) \\
n=-0.35\end{array}$} & \multicolumn{2}{|c|}{$\begin{array}{c}\text { Optimized } \\
\text { NGBM }(1,1) \\
n=-0.45, \alpha= \\
0.46\end{array}$} \\
\hline & & Prediction & $\begin{array}{l}\text { RPE } \\
(\%)\end{array}$ & Prediction & $\begin{array}{l}\text { RPE } \\
(\%)\end{array}$ \\
\hline 1994 & 20.3 & 20.3 & 0.00 & 20.3 & 0.00 \\
\hline 1995 & 29.5 & 29.503 & -0.01 & 29.482 & -0.06 \\
\hline 1996 & 31.1 & 30.812 & 0.92 & 30.894 & -0.66 \\
\hline 1997 & 37.2 & 36.064 & 3.05 & 36.708 & -1.32 \\
\hline 1998 & 38.2 & 43.917 & -14.97 & 45.385 & 18.81 \\
\hline 1999 & 57.5 & 54.534 & 5.16 & 57.189 & -0.54 \\
\hline MAPE (\%) & & & 4.82 & & 4.28 \\
\hline
\end{tabular}

TABLE 3: Results of optoelectronic application from the two models.

\begin{tabular}{|c|c|c|c|c|c|}
\hline \multirow[t]{2}{*}{ Year } & \multirow[t]{2}{*}{ Actual data } & \multicolumn{2}{|c|}{$\begin{array}{c}\operatorname{NGBM}(1,1), \\
n=0.7\end{array}$} & \multicolumn{2}{|c|}{$\begin{array}{c}\text { Optimized } \\
\operatorname{NGBM}(1,1) \\
n=0.91, \alpha=0.46\end{array}$} \\
\hline & & Prediction & $\begin{array}{l}\mathrm{RPE} \\
(\%)\end{array}$ & Prediction & RPE (\%) \\
\hline 1994 & 2.2 & 2.2 & 0.00 & 2.2 & 0.00 \\
\hline 1995 & 3.2 & 3.193 & 0.23 & 3.053 & -4.60 \\
\hline 1996 & 4.2 & 4.545 & -8.21 & 4.650 & 10.71 \\
\hline 1997 & 6 & 5.577 & 7.04 & 5.839 & -2.68 \\
\hline 1998 & 6.4 & 6.242 & 2.47 & 6.412 & 0.18 \\
\hline 1999 & 6.4 & 6.564 & -2.57 & 6.400 & 0.00 \\
\hline MAPE (\%) & & & 4.10 & & 3.64 \\
\hline
\end{tabular}

TABle 4: China's GDP from 2007 to 2018 (unit: $10^{12}$ yuan).

\begin{tabular}{cc}
\hline Year & Real data \\
\hline 2012 & 53.85 \\
2013 & 59.30 \\
2014 & 64.12 \\
2015 & 68.59 \\
2016 & 74.01 \\
2017 & 82.07 \\
2018 & 90.03 \\
\hline
\end{tabular}

from the five models are quite ideal, within the range $[-0.45 \%, 0.24 \%]$. Those of the five model are, however, generally more than $1 \%$ in the testing period; despite this, the 
TABle 5: Parameters of the five grey models.

\begin{tabular}{lcccccc}
\hline Parameter & M1 & M2 & M3 & M4 & New model (1st rolling) & New model (2nd rolling) \\
\hline$a$ & -0.070 & -0.070 & -0.067 & -0.067 & -0.067 & -0.112 \\
$b$ & 51.195 & 51.195 & 49.195 & 49.197 & 49.197 & 84.535 \\
$n$ & 0.01 & 0.01 & 0.02 & 0.02 & 0.02 & -0.10 \\
$a$ & 0.5 & 0.5 & 0.497 & 0.496 & 0.496 & 0.494 \\
\hline
\end{tabular}

TABLE 6: Results of simulation and prediction from the five grey models.

\begin{tabular}{ccccccc}
\hline Year & Actual data & M1 & M2 & M3 & M4 & New model \\
\hline 2012 & 53.85 & & & & & \\
2013 & 59.30 & 59.306 & 59.316 & 59.283 & 59.280 & 59.280 \\
2014 & 64.12 & 63.914 & 63.924 & 64.026 & 64.032 & 64.032 \\
2015 & 68.59 & 68.753 & 68.764 & 68.890 & 68.906 & 68.906 \\
2016 & 74.01 & 73.893 & 73.904 & 73.994 & 74.021 & 74.021 \\
2017 & 82.07 & 79.376 & 79.388 & 79.393 & 79.433 & $\begin{array}{c}79.433 \\
(1 \text { st rolling) }\end{array}$ \\
2018 & 90.03 & 85.237 & 85.250 & 85.128 & 85.183 & $\begin{array}{c}90.571 \\
\text { (2nd rolling) }\end{array}$ \\
\hline
\end{tabular}

TABLE 7: Relative error values of China's GDP data by the five grey models (\%).

\begin{tabular}{lccccc}
\hline Year & M1 & M2 & M3 & M4 & New model \\
\hline 2012 & 0 & 0 & 0 & 0 & 0 \\
2013 & 0.02 & 0.03 & -0.02 & -0.03 & -0.03 \\
2014 & -0.33 & -0.32 & -0.16 & -0.15 & -0.15 \\
2015 & 0.22 & 0.24 & -0.42 & -0.45 & -0.45 \\
2016 & -0.15 & -0.14 & -0.02 & 0.02 & 0.02 \\
2017 & -3.28 & -3.27 & -3.26 & -3.21 & -3.21 \\
2018 & -5.32 & -5.31 & -5.45 & -5.38 & $\mathbf{0 . 6 0}$ \\
MAPE & 1.55 & 1.54 & 1.48 & 1.45 & $\mathbf{0 . 6 5}$ \\
\hline
\end{tabular}

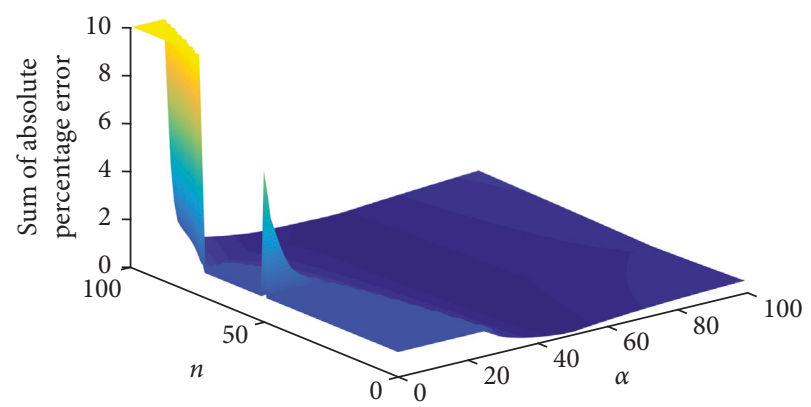

FIGURE 2: The track of simultaneously seeking the parameters $n$ and $\alpha$ in optoelectronic application.

prediction performance of the five models are still considered excellent, which can be referred in Table 1. It means the $\operatorname{NGBM}(1,1)$ model has a good performance on predicting the small sample time sequences with nonlinear characteristics.

We can further obverse that the MAPE of M1, M2, M3, M4, and new model are $1.55 \%, 1.54 \%, 1.48 \%, 1.45 \%$, and $0.65 \%$, respectively; this means optimizing the initial condition or optimizing the background values of the original $\operatorname{NGBM}(1,1)$ model can enhance the prediction accuracy of the original $\operatorname{NGBM}(1,1)$ model, while optimizing both parts at the same time has a better performance. The principle of new information priority is also seen by comparing the

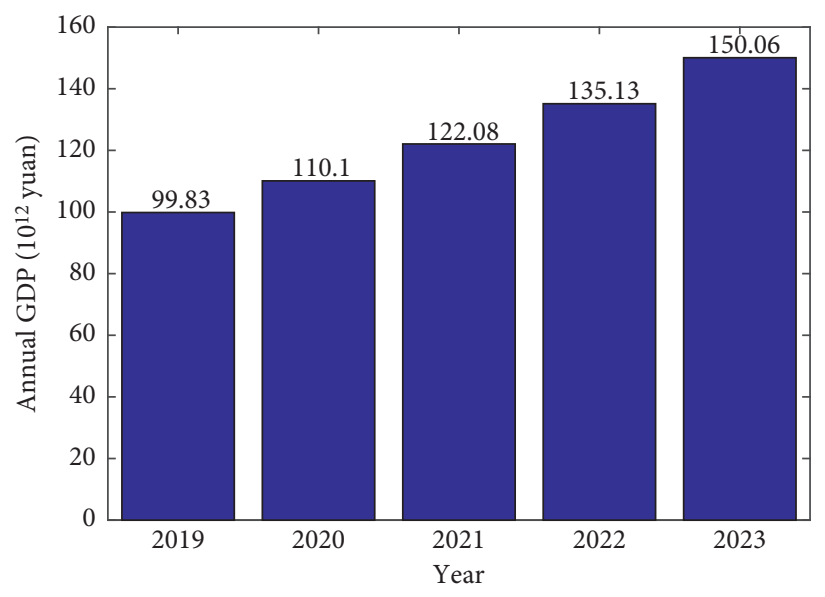

FIgURE 3: The predicted values of China's GDP from 2019 to 2023 by the optimized NGBM $(1,1)$ model.

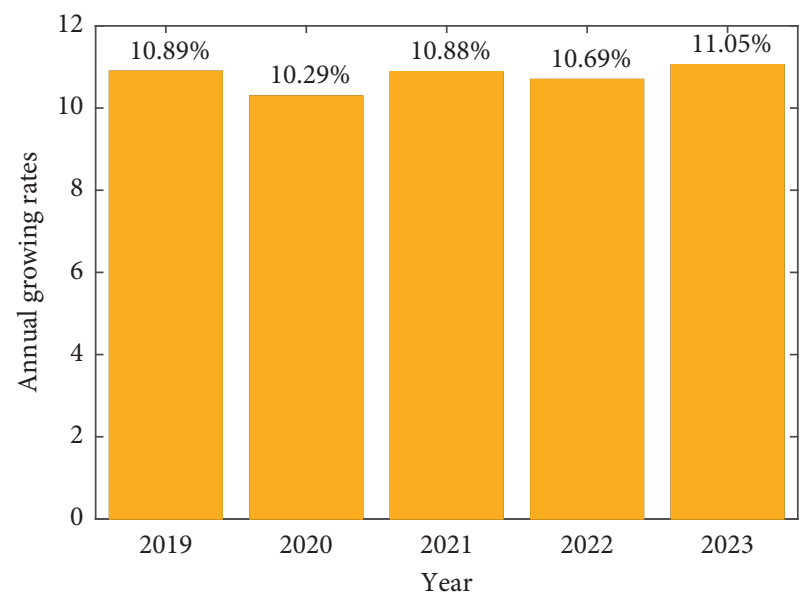

FIGURE 4: Increasing rates of annual GDP from 2019 to 2023.

MAPE of M4 and that of the new model. The computational results show the values predicted by the optimized $\operatorname{NGBM}(1,1)$ model are considerably closer to the actual values than those of the other models, and the optimized $\operatorname{NGBM}(1,1)$ model has the best prediction performance among these models.

5.3. Forecasting China's GDP from 2019 to 2023. Given the optimized $\operatorname{NGBM}(1,1)$ model shows the best performance in the sections above, we apply the optimized $\operatorname{NGBM}(1,1)$ model to forecast China's GDP data in the next five years. The predicted values and increasing rates are displayed in Figures 2 and 3.

It is not difficult to find from Figure 2 that annual GDP of China will continue to grow rapidly in the next five years. 
The annual GDP will grow to $1.56 \times 10^{14}$ yuan. According to Figures 3 and 4, we can observe that the increasing rates of annual GDP of China will be increasing and decreasing simultaneously. In summary, China's GDP will continue to grow steadily. At the same time, we must also see that the economic operation is stable and changeable, and the external environment is complicated and severe. The economy is facing a downward pressure. The problems in progress must be addressed in a targeted manner.

\section{Conclusion and Future Work}

In this paper, a novel optimized NGBM $(1,1)$ model has been proposed to forecast the annual GDP of China. The numerical results of simulation and real example have implied that the optimized $\operatorname{NGBM}(1,1)$ model has more excellent performance on forecasting the annual GDP than the other commonly used models. Then, the main conclusions are listed as follows.

(1) The novel optimized model can be considered as an extension of the $\operatorname{NGBM}(1,1)$ model, but the modeling procedure differs from that of the $\operatorname{NGBM}(1,1)$ model. Instead, we take the latest item of 1-AGO sequence as the initial condition, according to the principle of new information before using. Secondly, the parameter in the background value is automatically determined by minimizing the sum of absolute percentage errors. Finally, the model based on the rolling mechanism is established to further increase prediction precision of the grey model.

(2) As the optimized NGBM $(1,1)$ model shows the best performance among the existing models, we apply it to forecast the annual GDP of China in the next five years; it is obviously believed that the annual GDP will increase in the next few years, and the increasing rate also demonstrates the annual GDP will grow steadily.

However, there still exist some issues which should be discussed and solved in the future work, for example, it should be noticed that the size of sample time sequence used for predictive modeling is artificially defined in this paper; however, it is studied that a changeable sample size has a different effect on the grey system model [34]; therefore, how to scientifically determine the sample size should be discussed in future work. In addition, it can be obviously seen that the annual GDP in China is influenced by other factors, such as consumption, investment, and export-import, which are changeable and complex; in this paper, the modeling procedure of the optimized model is based on the sample time sequence themselves, that is, other information that cannot be ignored is not effectively used; therefore, the multivariate grey models will be concentrated in the future work.

\section{Data Availability}

The data used to support the findings of this study are deposited at http://www.stats.gov.cn/english/.

\section{Conflicts of Interest}

The authors declare that there are no conflicts of interest regarding the publication of this paper.

\section{Acknowledgments}

Tao Zhang's research was supported by the National Natural Science Foundation of China (Grant nos. 11861014 and 11561006) and Natural Science Foundation of Guangxi (2018JJA110013). Chengli Zheng's research was supported by the Humanities and Social Science Planning Fund from Ministry of Education (16YJAZH078), the Fundamental Research Funds for the Central Universities of China (Grant nos. CCNU 19TS062, CCNU19A06043, and CCNU19TD006), and the raising initial capital for High-Level Talents of Central China Normal University (30101190001).

\section{References}

[1] J. Buckley and I. James, "Linear regression with censored data," Biometrika, vol. 66, no. 3, pp. 429-436, 1979.

[2] M. Roozbeh, M. Arashi, and H. A. Niroumand, "Semiparametric ridge regression approach in partially linear models," Communications in Statistics-Simulation and Computation, vol. 39, no. 3, pp. 449-460, 2010.

[3] A. R. Gallant and J. J. Goebel, "Nonlinear regression with autocorrelated errors," Journal of the American Statistical Association, vol. 71, no. 356, pp. 961-967, 1976.

[4] S. E. Said and D. A. Dickey, "Testing for unit roots in autoregressive-moving average models of unknown order," Biometrika, vol. 71, no. 3, pp. 599-607, 1984.

[5] N. An, W. Zhao, J. Wang, D. Shang, and E. Zhao, "Using multi-output feedforward neural network with empirical mode decomposition based signal filtering for electricity demand forecasting," Energy, vol. 49, pp. 279-288, 2013.

[6] T. Papadimitriou, P. Gogas, and E. Stathakis, "Forecasting energy markets using support vector machines," Energy Economics, vol. 44, pp. 135-142, 2014.

[7] J.-L. Deng, "Control problems of grey systems," Systems \& Control Letters, vol. 1, no. 5, pp. 288-294, 1982.

[8] B. Zeng and S. Liu, "A self-adaptive intelligence gray prediction model with the optimal fractional order accumulating operator and its application," Mathematical Methods in the Applied Sciences, vol. 40, no. 18, pp. 7843-7857, 2017.

[9] B. Zeng and C. Li, "Forecasting the natural gas demand in China using a self-adapting intelligent grey model," Energy, vol. 112, pp. 810-825, 2016.

[10] N. Xie, B. Su, and N. Chen, "Construction mechanism of whitenization weight function and its application in grey clustering evaluation," Journal of Systems Engineering and Electronics, vol. 30, no. 1, pp. 121-131, 2019.

[11] B. Wei, N. Xie, and A. Hu, "Optimal solution for novel grey polynomial prediction model," Applied Mathematical Modelling, vol. 62, pp. 717-727, 2018.

[12] X. Ma and Z.-B. Liu, "The kernel-based nonlinear multivariate grey model," Applied Mathematical Modelling, vol. 56, pp. 217-238, 2018.

[13] X. Ma, M. Xie, W. Wu, B. Zeng, Y. Wang, and X. Wu, "The novel fractional discrete multivariate grey system model and its applications," Applied Mathematical Modelling, vol. 70, pp. 402-424, 2019. 
[14] W. Wu, X. Ma, Y. Wang, Y. Zhang, and B. Zeng, "Research on a novel fractional $\operatorname{GM}(\alpha, n)$ model and its applications," Grey Systems: Theory and Application, vol. 9, no. 3, pp. 356-373, 2019.

[15] B. Zeng, H. Duan, and Y. Zhou, "A new multivariable grey prediction model with structure compatibility," Applied Mathematical Modelling, vol. 75, pp. 385-397, 2019.

[16] B. Zeng and C. Li, "Improved multi-variable grey forecasting model with a dynamic background-value coefficient and its application," Computers \& Industrial Engineering, vol. 118, pp. 278-290, 2018.

[17] T.-L. Tien, "A research on the prediction of machining accuracy by the deterministic grey dynamic model $\operatorname{DGDM}(1,1,1)$," Applied Mathematics and Computation, vol. 161, no. 3, pp. 923-945, 2005.

[18] N.-M. Xie and S.-F. Liu, "Discrete grey forecasting model and its optimization," Applied Mathematical Modelling, vol. 33, no. 2, pp. 1173-1186, 2009.

[19] S. Liu, Y. Yang, and J. Forrest, Grey Data Analysis, Springer, Berlin, Germany, 2017.

[20] C.-I. Chen, "Application of the novel nonlinear grey Bernoulli model for forecasting unemployment rate," Chaos, Solitons \& Fractals, vol. 37, no. 1, pp. 278-287, 2008.

[21] Z.-X. Wang, K. W. Hipel, Q. Wang, and S.-W. He, "An optimized $\operatorname{NGBM}(1,1)$ model for forecasting the qualified discharge rate of industrial wastewater in China," Applied Mathematical Modelling, vol. 35, no. 12, pp. 5524-5532, 2011.

[22] L. Pei, Q. Li, and Z. Wang, "The nls-based nonlinear grey Bernoulli model with an application to employee demand prediction of high-tech enterprises in China," Grey Systems: Theory and Application, vol. 8, no. 2, pp. 133-143, 2018.

[23] Z.-X. Wang, "A weighted non-linear grey Bernoulli model for forecasting non-linear economictime series with small data sets," Economic Computation \& Economic Cybernetics Studies \& Research, vol. 51, no. 1, 2017.

[24] L.-L. Pei and Q. Li, "Forecasting quarterly sales volume of the new energy vehicles industry in China using a data grouping approach-based nonlinear grey Bernoulli model," Sustainability, vol. 11, no. 5, p. 1247, 2019.

[25] C.-I. Chen, P.-H. Hsin, and C.-S. Wu, “Forecasting Taiwan's major stock indices by the Nash nonlinear grey Bernoulli model," Expert Systems with Applications, vol. 37, no. 12, pp. 7557-7562, 2010.

[26] X. Liu and N. Xie, "A nonlinear grey forecasting model with double shape parameters and its application," Applied Mathematics and Computation, vol. 360, pp. 203-212, 2019.

[27] X. Ma, Z. Liu, and Y. Wang, "Application of a novel nonlinear multivariate grey Bernoulli model to predict the tourist income of China," Journal of Computational and Applied Mathematics, vol. 347, pp. 84-94, 2019.

[28] H. Duan, X. Xiao, and L. Pei, "Forecasting the short-term traffic flow in the intelligent transportation system based on an inertia nonhomogenous discrete gray model," Complexity, vol. 2017, Article ID 3515272, 16 pages, 2017.

[29] Z.-X. Wang and L. Qin, "Modelling the nonlinear relationship between $\mathrm{co} 2$ emissions and economic growth using a pso algorithm-based grey verhulst model," Journal of Cleaner Production, vol. 207, pp. 214-224, 2019.

[30] L. Wu, G. Huang, J. Fan, F. Zhang, X. Wang, and W. Zeng, "Potential of kernel-based nonlinear extension of arps decline model and gradient boosting with categorical features support for predicting daily global solar radiation in humid regions," Energy Conversion and Management, vol. 183, pp. 280-295, 2019.
[31] J. Zhou, R. Fang, Y. Li, Y. Zhang, and B. Peng, "Parameter optimization of nonlinear grey Bernoulli model using particle swarm optimization," Applied Mathematics and Computation, vol. 207, no. 2, pp. 292-299, 2009.

[32] S. Ding, K. W. Hipel, and Y.-G. Dang, "Forecasting China's electricity consumption using a new grey prediction model," Energy, vol. 149, pp. 314-328, 2018.

[33] X. Xiao, J. Yang, S. Mao, and J. Wen, "An improved seasonal rolling grey forecasting model using a cycle truncation accumulated generating operation for traffic flow," Applied Mathematical Modelling, vol. 51, pp. 386-404, 2017.

[34] L. Wu, S. Liu, L. Yao, and S. Yan, "The effect of sample size on the grey system model," Applied Mathematical Modelling, vol. 37, no. 9, pp. 6577-6583, 2013. 


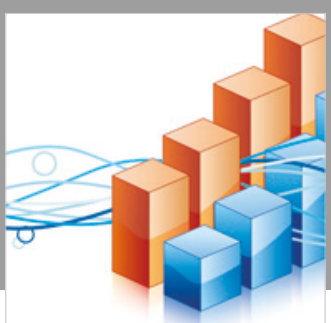

Advances in

Operations Research

\section{-n-m}
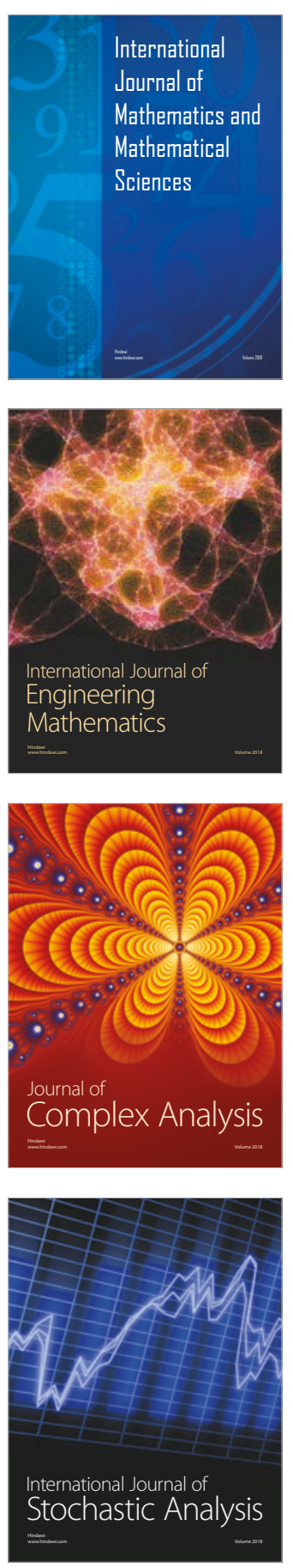
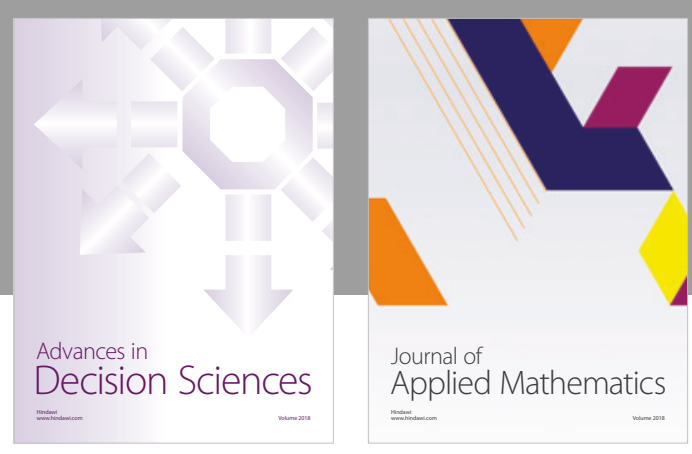

Journal of

Applied Mathematics
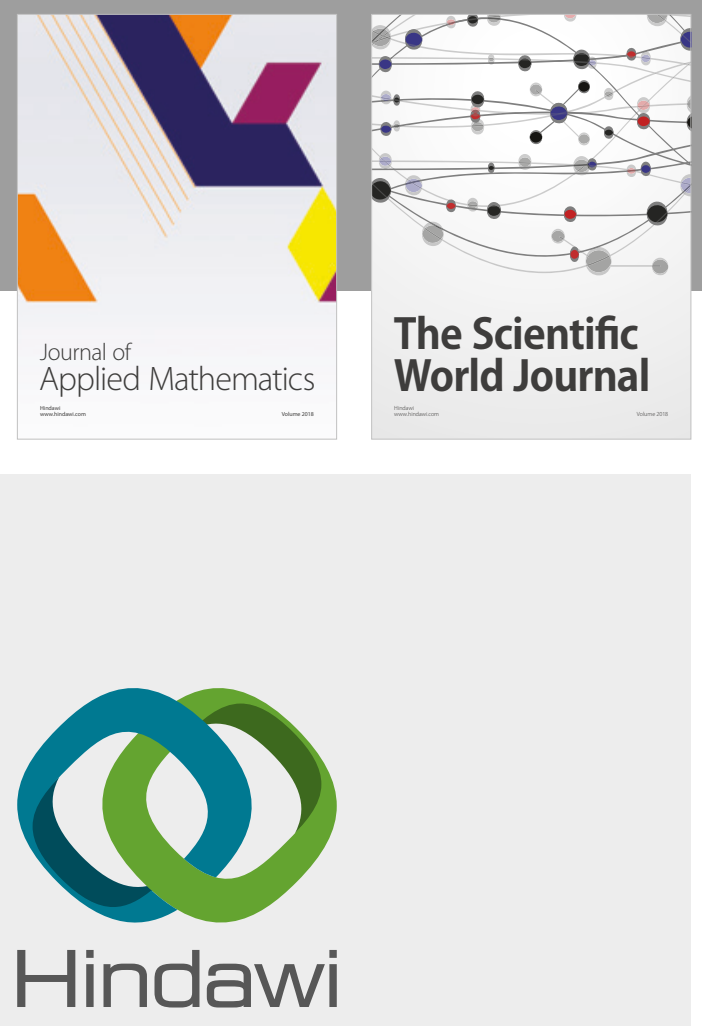

Submit your manuscripts at

www.hindawi.com

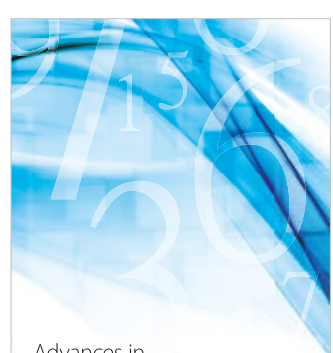

Advances in
Numerical Analysis
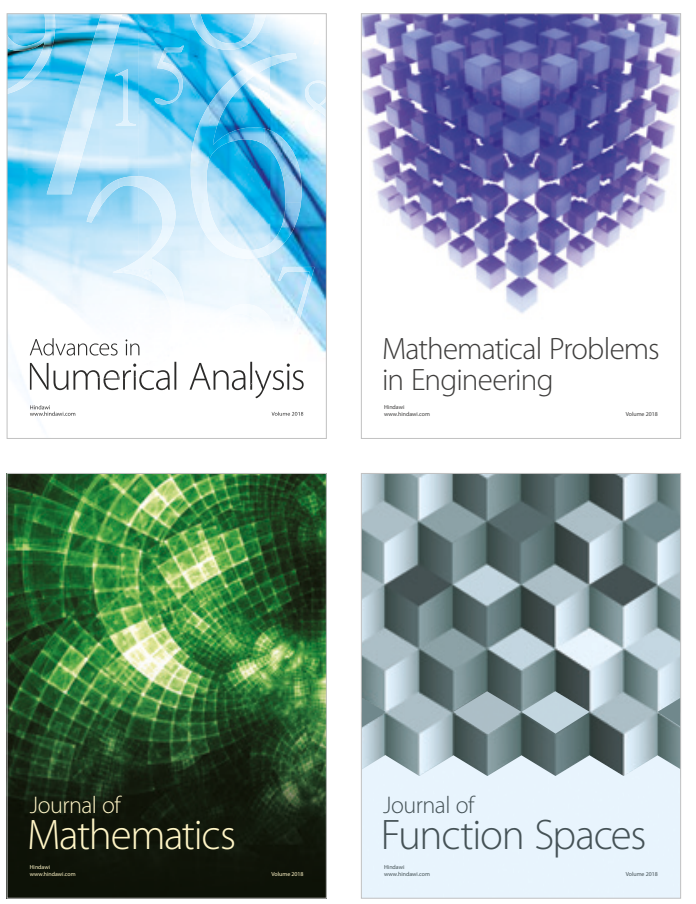

Mathematical Problems in Engineering

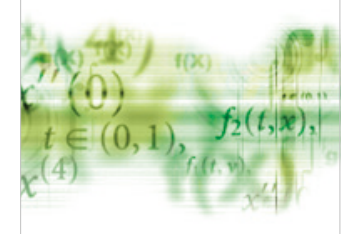

International Journal of

Differential Equations

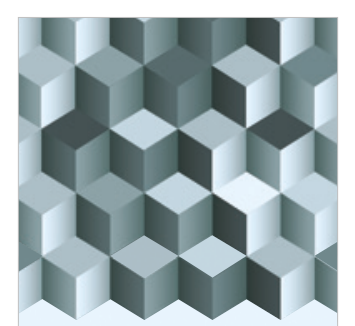

Journal of

Function Spaces
The Scientific

World Journal

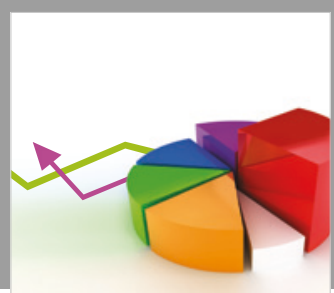

Journal of

Probability and Statistics
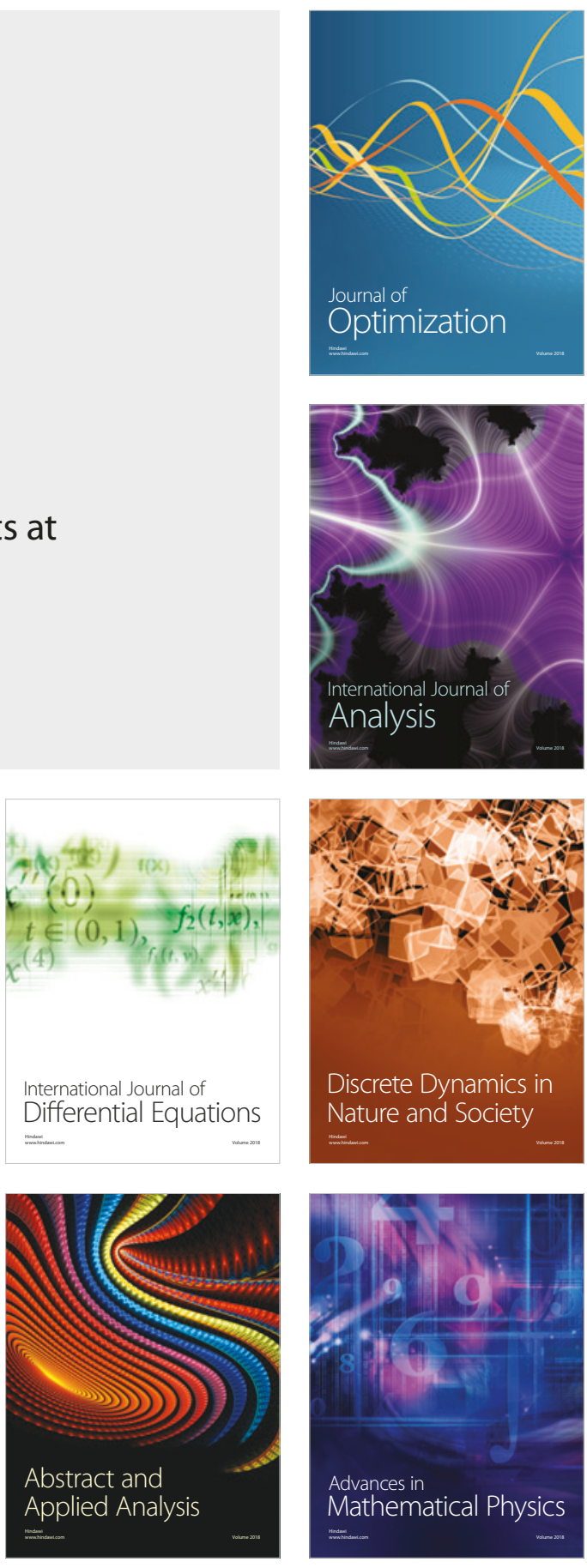